rev.relac.int.estrateg.segur.13(2):97-121,2018

\title{
¿Soft power o hard power? Reflexiones teóricas sobre la política exterior brasileña*
}

\author{
Andrés Peña Galindo**
}

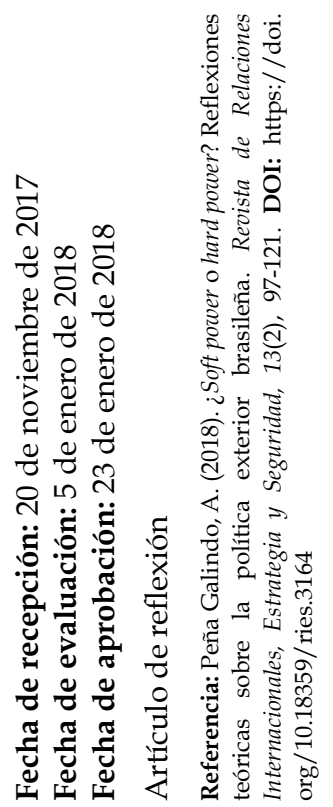

\section{Resumen}

Basado en un análisis neorrealista y en contraposición a los planteamientos del soft power, este artículo estudia la política exterior brasileña respecto a posibles diseños hegemónicos regionales. La investigación examina avances cruciales en el poder militar de Brasil, pero también tiene en cuenta la capacidad demográfica y económica brasileña y su posibilidad de desarrollar armas nucleares, como elementos condicionantes para equilibrar el poder en el escenario global. El análisis se contextualiza en la estructura sistémica internacional, donde Estados Unidos, como la gran potencia, debe impedir que otros Estados adquieran poder.

* Tras una investigación, de aproximadamente dos años, en el marco de la Maestría en Asuntos Internacionales de la Universidad Externado de Colombia, se materializaron dos resultados: la publicación del libro Brasil. ¿Hegemonía a pesar de todo?, en coautoría con el doctor en Seguridad Internacional y Defensa Florent Frasson-Quenoz, y el presente artículo como una respuesta crítica al planteamiento del soft power para entender la política exterior brasileña.

** Doctorando en Estudios Políticos e Internacionales de la Universidad del Rosario; magíster en Asuntos Internacionales de la Universidad Externado de Colombia. Correo electrónico: andresar.pena@ urosario.edu.co. 
Palabras clave: Brasil; hegemonía; poder; realismo ofensivo; Suramérica.

\title{
Soft Power or Hard Power? Theoretical Reflections on Brazilian Foreign Policy
}

\begin{abstract}
Based on a neorealist analysis, and contrary to the soft power approach, this article studies Brazilian foreign policy with regards to potential regional hegemonic designs. The research examines crucial developments in the military power of Brazil but also considers its demographic and economic capacity and its potential to develop nuclear weapons as determining factors to balance power on a global scale. The analysis is contextualized in the international systemic structure, where the United States as the great power should prevent other states from gaining power.
\end{abstract}

Keywords: Brazil; hegemony; offensive realism; power; South America.

\section{Soft power ou hard power? Reflexões teóricas sobre a política exterior brasileira}

\section{Resumo}

Baseado em uma análise neorrealista e em contraposição as abordagens do soft power, este artigo estuda a política exterior brasileira no que se refere a possíveis desenhos hegemônicos regionais. A pesquisa examina avanços cruciais no poder militar do Brasil, mas também leva em conta a capacidade demográfica e econômica brasileira e sua possibilidade de desenvolver armas nucleares, como elementos condicionantes para equilibrar o poder no cenário global. A análise se contextualiza na estrutura sistêmica internacional, onde os Estados Unidos, como a grande potência, deve impedir que outros Estados adquiram poder.

Palavras-chave: Brasil; hegemonia; poder; realismo ofensivo; América do Sul. 


\section{Introducción}

Desde las luchas de independencia de los Estados sudamericanos, Brasil se ha diferenciado de sus pares regionales. En un principio, sus relaciones estaban primordialmente guiadas por Europa y Estados Unidos como una táctica de seguidismo (bandwagoning) ${ }^{1}$ frente a los Estados más desarrollados, aunque esporádicamente se dirigía al Cono Sur (Candeas, 2012). Durante el siglo XX, consagró un progreso importante con miras a establecerse como el país más poderoso de su región, al punto de que Stefan Zweig lo catalogó como el "país del futuro" (2012). Este objetivo se estuvo cumpliendo hasta la década de los ochenta, cuando entró en una importante crisis económica que lo obligó a replantear sus tácticas.

Dicha crisis tuvo lugar en la que, hasta hoy, ha sido la última dictadura en el país sudamericano (1964-1985), e impulsó la disolución de esta. Simultáneamente al proceso de democratización en Brasil, el sistema internacional sobrellevó un importante cambio: el orden bipolar dejó de existir. Según John Mearsheimer, lo anterior implicó la conformación de una estructura multipolar desbalanceada (2001, p. 339). Esta nueva polaridad en el sistema condujo a Estados Unidos a re- tirarse de ciertas regiones del mundo donde hacía presencia (Buzan y Waever, 2003).

Más allá del contexto interno y externo, desde mediados de la década de los ochenta, Brasil viene adquiriendo un mayor protagonismo a escala internacional. La infraestructura alcanzada en términos de poderío militar le permitía ser visto como un Estado en búsqueda de autonomía material. Para la época, Ethan Kapstein consideró que estaba encaminado a adquirir independencia armamentista (1990), lo que, en términos del realismo estructural en general y el ofensivo en particular, tiene sentido, debido a que para dicha teoría los Estados pugnan por ser los más poderosos —en el ámbito armamentistaen el sistema internacional.

En Suramérica, Brasil buscó adoptar un papel de liderazgo como contrapeso a Estados Unidos. Militares como el almirante argentino Juan Guglialmelli alertaron una especie de destino manifiesto, impulsado por Brasil, en la región ${ }^{2}$ (Hepple, 1986, p. 585). Carlos Poggio Teixeira trajo a colación una frase de Henry Kissinger para evidenciar tal contrapeso: "[Existe] una competencia táctica entre Brasil y los Estados Unidos en Sur América desde el final de la Guerra Fría" (2014, p. 130). Ade-

1 Según John Mearsheimer (2001, p. 139), la finalidad de esta táctica es no convertirse en el enemigo de un Estado más poderoso y, así, evitar ser destruido por este, ya que el Estado débil cede ante las peticiones del poderoso. El investigador estadounidense aclara que tal táctica no debe ser la opción principal porque concede poder, y eso, en el sistema internacional, resulta siendo contraproducente.

2 No obstante, poco logró con la denuncia, ya que Argentina no ha conseguido establecerse como un competidor regional para Brasil (Vitelli, 2016). 
más, debido a la prioridad que Estados Unidos les ha dado a otras zonas geográficas del mundo - especialmente después de los atentados del 11 de septiembre de 2001-, Brasil ha gozado de un margen de maniobra ampliado en su región (Pastrana Buelvas y Vera Piñeros, 2012).

Para Monica Hirst (2015, p. 361), Brasil ha buscado preponderancia a través de optar por una nueva arquitectura del sistema. El argumento de la profesora se sustenta en lo que ella llama las cuatro aspiraciones de Brasil para conseguir reconocimiento internacional, que son: 1) más influencia en el rediseño de una arquitectura global multilateral; 2) mayor responsabilidad en escenarios de crisis humanitarias, posconflicto y desastres naturales acorde a las instituciones y normas multilaterales; 3) agenda ampliada para la cooperación sur-sur, y 4) un rol regional preponderante, marcado por asuntos de paz, estabilidad y desarrollo sostenible.

Hirst señala que Brasil busca abrir los espacios, mediante mayores relaciones y respeto de las instituciones y normas internacionales, para contribuir a un reordenamiento del sistema internacional. Como evidencia, la autora resalta que el Estado sudamericano logró hacer parte de la ampliación del G-8 al G-20 y de grupos de Estados emergentes, como Brasil, Rusia, India, China y Sudáfrica (BRICS) o el Foro de Diálogo India, Brasil y Sudáfrica (IBSA). En el mismo sentido, Mathilde Chatin (2016) argumenta que Brasil hace uso del soft power y no de las capacidades militares para adquirir un papel de liderazgo mundial. Sin embargo, el actuar de Brasil no responde únicamente al soft power, sino también a una estrategia para crecer militarmente en un sistema cuya polaridad está desequilibrada en favor de un solo agente.

El objetivo de este artículo es, entonces, analizar las medidas adoptadas por Brasil, desde 1985 hasta 2012, para establecerse como gran potencia a nivel regional, a partir de los enunciados de John Mearsheimer. Tal aproximación teórica nació — como el mismo autor señala - en respuesta a los planteamientos con los que se estudiaba la disciplina de las relaciones internacionales a lo largo de la década de los noventa (2001, p. 1), cuando diferentes analistas supusieron - tras el final de la Guerra Fría- la reducción de la anarquía en el sistema internacional, gracias a una mayor interdependencia de Estados y el establecimiento de instituciones supranacionales.

Mearsheimer (2001) argumenta: la competencia por la seguridad y la guerra entre las potencias no se eliminó de la política internacional; las organizaciones internacionales no son más que herramientas utilizadas por las grandes potencias para su beneficio; el Estado sigue siendo el actor preponderante en el sistema internacional; los discursos no necesariamente reflejan las verdaderas intenciones de los países, y el comportamiento está guiado por tres patrones: el miedo, el self-help (autoayuda) y la maximización de po- 
der. Como consecuencia, los Estados prestan atención a la distribución del poder y se esfuerzan por maximizar el propio, teniendo en cuenta - como Estados racionales- ${ }^{3}$ los costos y riesgos, contra los beneficios de sus acciones, debido a que cada Estado es el único responsable por su supervivencia en un sistema internacional sin un ente superior que regule el comportamiento de los actores.

Para sobrevivir, los Estados cuentan con dos tipos de poder: el latente y el real. El primero hace referencia a los insumos necesarios para la estructuración del segundo y está conformado por el territorio, la población y la economía. El segundo —que es la misma capacidad militar- se cuantifica en el escenario internacional para conocer la fuerza del Estado (2001, p. 55). Los que cuentan con el mayor poder real son aquellos que podrán posicionarse como hegemones $^{4}$ (p. 29) y, así, moldear el comportamiento de los demás para su beneficio. Existe un tercer tipo de poder que resulta definitivo: el nuclear. Debido a su capacidad destructiva casi instantánea, el Estado que posea superioridad en este campo será el hegemón, pero al existir otras potencias con capacidades atómicas, la superioridad nuclear quedará anulada y los otros poderes — real y latentemantendrán su importancia (p. 129).
Es importante resaltar que Mearsheimer describe los comportamientos de las grandes potencias. Así que el ejercicio que se realiza con esta investigación tiene un carácter novedoso, pues aplica una teoría estructural, pensada para potencias mundiales a una potencia de segundo rango, como lo es Brasil. La intención es, por un lado, evidenciar que una potencia media como el Estado suramericano cumple con patrones de comportamiento de un Estado racional, expuestos por el realismo ofensivo ${ }^{5}, y$, por otro, generar argumentos en contra de los planteamientos que aseguran que Brasil recurre al soft power para adquirir un nuevo rol en el sistema internacional.

A continuación, se abordará el poder real de Brasil; su poder latente será tratado en la segunda parte; su poder nuclear, en la tercera, $y$, finalmente, se desarrollarán sus posibilidades dentro de la estructura actual del sistema internacional.

\section{El poder real brasileño}

Mearsheimer aclara que el poder real es el poder esencial que tienen los Estados para configurarse como una gran potencia. Entendiendo tal importancia, Brasil se ha preocupado por ganar independencia en ese aspecto para adquirir ventaja a nivel regional. Desde

\footnotetext{
3 Para profundizar en este tema, véase Mearsheimer (2009).

4 En términos de Morton Kaplan (1957), un Estado hegemónico — a nivel mundial— es aquel que mantiene relaciones dominantes con otros actores y el sistema en general.

5 Vale la pena señalar que, para dicha teoría, no hace falta hacer uso de la fuerza adquirida. En la mayoría de casos, contar con un poder militar superior es suficiente para disuadir a los demás actores, en pro de mayores beneficios para el Estado que cuenta con dicho poderío.
} 
la década de los setenta, implementó acuerdos de producción bajo licencia, coproducción y transferencia tecnológica con Estados más desarrollados en la generación de armamento (Vargas Vergnaud, 2004). Esos acuerdos permitieron la creación de empresas propias, capaces de producir material militar. En este sentido, y desde ese momento, "Brasil pasó a ser así el único país latinoamericano con la autosuficiencia necesaria para sostener un conflicto bélico sin tener que recurrir a equipos militares importados" (Acuña, Smith y Wolfson 1994, p. 363). Gracias a la independencia conseguida, la potencia sudamericana logró, además de establecerse en la región como el mayor productor e importador de instrumentos defensivos (Sipri, 1978), transformarse en uno de los países en vía de desarrollo con mayor exportación de armamentos ${ }^{6}$ (Mercado-Jarrín, 1982, p. 7).

No obstante, para finales del siglo $\mathrm{XX}$, la crisis económica y la ausencia de una amenaza regional debilitaron el impulso brasileño en el campo del poder real, a tal punto que el gasto militar se redujo del $1,9 \%$ del producto interno bruto (PIB) en 1990 al 1,3\% en 1999 (González, 2003). Esto significa que como el PIB de Brasil de
1990 alcanzó los 571.293 millones de dólares (valor constante, base 1995) y el de 1999 correspondió a 711.487 millones de dólares (valor constante, base 1995) (Fresard y Olarte, 2001, p. 153), su gasto militar se disminuyó en 1.605,236 millones de dólares sobre este mismo periodo. Ya en el siglo XXI, el escenario volvió a cambiar. La recuperación de su poder latente ${ }^{7}$, el aumento del poderío militar de algunos Estados vecinos y la amenaza de un incremento de la presencia estadounidense en la región llevaron a Brasil a tomar nuevas políticas con respecto a su capacidad militar (Calle, 2008).

La preocupación frente a una mayor presencia estadounidense en la región se evidenció en la estrategia de defensa, emanada por el gobierno brasileño en 2008; allí se resalta la importancia de contar con unas fuerzas militares aptas para responder a cualquier amenaza externa, proveniente, esencialmente, de una gran potencia ${ }^{8}(\mathrm{Mi}-$ nisterio de Defensa de Brasil, 2008). Además:

[E]n enero de 2007, la principal agencia de inteligencia del Brasil dio a conocer un informe en donde advertía y criticaba sobre el creciente "cerco" que viene montando

6 Según datos del Stockholm International Peace Research Institute (Sipri), en el periodo de 1979-1981, Brasil, con el 46,5\%, fue el Estado no desarrollado que más armamento pesado exportó (1982, p. 188).

7 Según cifras del Fondo Monetario Internacional (2010), Brasil alcanzó en la primera década del siglo xxı el octavo lugar en cuanto a la economía más poderosa a nivel mundial.

8 Es importante aclarar que los Estados pueden usar el discurso como disuasión. No necesariamente refleja las verdaderas intenciones, preocupaciones o planes. Pero, en este caso particular, se resalta un discurso de esta naturaleza porque, más allá de las verdaderas preocupaciones del Estado brasileño, es una invitación a un empoderamiento militar, que es cuestión vital, y la más relevante, según el realismo ofensivo. 
Washington con la instalación de bases de diferentes tamaños en países fronterizos al Brasil, tales son los casos de Colombia, zonas del Caribe, Ecuador, Paraguay y Perú. (Calle, 2008, p. 175)

Simultáneamente, entre 1993 y 2002 en la región hubo un incremento del $20 \%$ en el gasto militar (Sipri, 2003). En este sentido, como sostiene Mearsheimer, la priorización del poder real se da por la intención de conseguir, en un primer momento, hegemonía regional (2001, p. 5). Así las cosas, los países deben preocuparse por el balance de poder en su región antes que estudiar su proyección mundial.

En este aspecto, la atención recae principalmente sobre el incremento del poderío militar de Chile, Venezuela y Colombia. Son estos Estados los que han demostrado un importante nivel de adquisición de poderío real configurándose en los más capacitados para equilibrar el poder en la región. Pero más allá de sus gastos en defensa, los tres tienen limitantes que les impiden alcanzar una eventual posición hegemónica. Chile es reducido en territorio y población, al igual que en número de militares (Bertonha, 2010a, p. 112), mientras que Colombia y Venezuela no organizan sus fuerzas para posibles proyecciones - elemento esencial para las grandes potencias-, sino que se preocupan por el orden interno y la defensa ante intromisiones extranjeras, respectivamente. Aún con estos condicionantes, era notoria una reactivación militar en la región, reactivación en la que Brasil no se quedó atrás.

Para 2002, el nivel en que se encontraba el armamento brasileño era preocupante. Aunque su inversión en poderío militar era la decimocuarta más alta del mundo (Sipri, 2003) y contaba con un importante número de militares activos, estos no estaban soportados en buen entrenamiento o en maquinaria moderna (Bertonha, 2008). Esta preocupación se materializó en 2007 cuando, a través de su Ministerio de Defensa, Brasil declaró necesario el fortalecimiento del instrumento militar. Esta declaración le otorgó una gran importancia al desarrollo de capacidades de guerra, ante lo que desde el mismo ministerio se señaló como "la posibilidad de injerencia de alguna potencia extra-regional". Además, el ministro resaltó, por una parte, al Amazonas y al Atlántico Sur como prioridades estratégicas (Costa, 2007), y, por otra, a la cibernética, la energía nuclear y el sector espacial como temas fundamentales. Siguiendo los lineamientos establecidos por el ministro de defensa, se creó un comité para la elaboración de la Estrategia Nacional de Defensa.

Pensar en una invasión estadounidense - sin lugar a duda es la potencia extrarregional a la que el Ministerio de Defensa brasileño hace referenciaes, cuanto menos, difícil. El Estado norteamericano tiene preocupaciones más grandes en otros hemisferios, y Brasil es visto como un aliado regio- 
nal que, aunque compite en varios aspectos, está lejos de ser una amenaza para el balance de poder actual (Poggio Teixeira, 2014). Sin embargo, como lo expone Mearsheimer (2001), este tipo de conjeturas son elementos que abren las puertas para un eventual incremento de poderío real. En ese sentido, la amenaza de una injerencia extrajera en el territorio sirvió como impulso para revitalizar el poderío militar, lo que le permitiría al Estado suramericano una mejor posición en el escenario internacional. Esto evidencia que las políticas del soft power resultan insuficientes ante dicho objetivo.

Puntualmente, para finales de la primera década del siglo XXI, la hoy potencia sudamericana anunció el mayor acuerdo militar de la historia del país desde la Guerra Fría (Goyzueta, 2009). Dicho acuerdo se realizó con Francia, una de las potencias militares de la época, con la intención de adquirir tecnología militar satelital, helicópteros Super Cougar para fabricar en el país suramericano y avances para el desarrollo de un submarino nuclear (Tokatlian, 2011, p. 148; Sipri, 2011, p. 171). "Según el Ministerio de Defensa francés, Brasil es su mejor cliente, ya que los contratos firmados entre Sarkozy y Lula suman unos 10.000 millones de dólares en el último quinquenio" (Uría Rodríguez, 2011, p. 16). Conjuntamente:

El tratado con Francia cumple con los objetivos militares y estratégicos de Brasil perfectamente. Le permite, geopolítica y simbólicamente, un nivel de distanciamiento del poder americano pero sin darle a Washington una causa para temer. Una asociación estratégica con Rusia o China despertaría una alerta en los americanos, pero una asociación con Francia no. (Bertonha, 2010b, p. 120)

Con lo anterior, Brasil ha establecido su industria militar como la más desarrollada de Suramérica (Argumosa Pila, 2011, p. 24) y ha incrementado su gasto en defensa; esto, según Daniel Flemes, le permite "asegurar su estatus de potencia militar dominante en la región" (2012, p. 41), y evita que la gran potencia del sistema internacional -Estados Unidos- frene o intervenga en su crecimiento. Es decir, Brasil encontró necesario reforzar su poder real para afirmar tanto la autonomía como su posición en la región (Duarte y Viana, 2008). Nuevamente, la importancia otorgada al sector militar deja en evidencia que las políticas del soft power no son herramienta suficiente para moldear la arquitectura del sistema internacional en favor de un Estado (Brasil, para este caso).

Con la intención de trascender la cooperación militar hacia una independencia en el mismo sector, Brasil ha generado un engranaje para el desarrollo científico y tecnológico de la defensa. Dicho desarrollo provee una interconexión entre universidades, centros de investigación y desarrollo, empresas de ingeniería, empresas industriales $y$, finalmente, empresas de servicio y productos de defensa. Igual- 
mente, el gobierno se ha encargado de crear y vincular al Estado empresas para la producción de material de defensa (Resdal, 2012, p. 155).

Las políticas mencionadas han permitido que Brasil se establezca como el Estado con mayor gasto militar en la región y uno de los mayores a nivel mundial (Gratius, 2008), lo que concuerda con sus intenciones enmarcadas en la Estrategia Nacional de Defensa, donde ratificó la importancia de la proyección internacional y de la capacidad de defensa frente a amenazas o agresiones a la soberanía. A propósito, ha venido ejerciendo una preparación de sus fuerzas militares para una eventual guerra asimétrica, en pro de tener la capacidad de respuesta frente a una potencia militarmente superior que tenga intereses en la región amazónica (Delgado, 2011). Como ya se señaló, el punto importante acá no es la posible —en verdad improbableinvasión extranjera; lo relevante es la revitalización del poder real.

El objetivo de obtener un lugar más importante en el sistema internacional desde la perspectiva del soft power limita el crecimiento material a una mayor participación en acciones coordinadas. Monica Hirst (2015, p. 366) reduce las capacidades militares brasileñas a su creciente contribución en misiones de mantenimiento de paz emanadas desde las Naciones Unidas.
Pero para hacer parte de este tipo de procedimientos no se necesita mayor capacidad de poder real. Muestra de lo anterior es que para 2005, Uruguay - lejos de ser uno de los Estados más poderosos en Suramérica- era el país de la región que más soldados aportaba a las operaciones de mantenimiento de paz (Zurbriggen, 2005). La importancia que Brasil le viene dando a las capacidades materiales responde a una lógica alejada del soft power.

Es posible afirmar que, al menos hasta principios de la segunda década del siglo XXI, Brasil se ha esforzado por contar con un poder real que responda a un interés por la protección integral de su territorio encaminado a configurarse en uno de los más fuertes del mundo, para obtener un estatus de país poderoso (Peña Galindo y Frasson-Quenoz, 2017). En palabras de Celso Amorim", "el fortalecimiento de la industria brasileña de material de defensa es uno de los ejes de nuestra estrategia nacional" (2012, p. 505). En términos generales, desde la propuesta de Mearsheimer, es claro que la potencia sudamericana cumple con las características de un Estado que pugna por establecerse, al menos, como hegemón regional, ya que usa su poder material en pro de un espacio mayor en el sistema internacional. Pero nada de ello sería posible sin la capacidad demográfica y económica: el poder latente.

9 Amorim fue ministro de Relaciones Exteriores de Brasil, durante dos periodos (1993-1995 y 2003-2010). La referencia acá citada es de 2012, cuando ejercía como ministro de Defensa. 


\section{Poderío económico, territorial y poblacional}

Mearsheimer reconoce la capacidad demográfica y económica del Estado como poder latente, que es necesario para soportar el poder real. En esa categoría, para 2012, Brasil era el octavo país con mayor PIB a nivel mundial, el quinto más grande del mundo y el sexto más poblado (CIA, 2013). En la actualidad, es el Estado con mayor cantidad de habitantes de América Latina y el Caribe (World Bank, 2005). Desde 1889, cuando se estableció como República, Brasil se ha preocupado por contar con un poder latente digno de una potencia mundial. A partir de los primeros años del siglo XX entró en disputa con sus vecinos en búsqueda de incrementar su territorio. De esa manera, y sin necesidad de utilizar su fuerza militar, consiguió expandirse firmando acuerdos con Perú y Bolivia en 1902 (Fernández Bengoechea, 2007), con Ecuador en 1904, con Venezuela en 1905 y con Colombia en 1907 (Da Silva, 2011, p. 8). Una vez conseguido ese objetivo, buscó una relación cercana con los países sudamericanos y un vínculo más grande, pero cuidadoso, con Estados Unidos (Fernández Bengoechea, 2007).

Ya durante la Guerra Fría, la hoy potencia sudamericana pujó por independencia frente a los dos bloques dominantes, principalmente para evitar que se le impidiera su desarrollo nuclear (Moniz Bandeira, 2006). Para la década de los setenta, Brasil conjugó en el Tratado de Cooperación
Amazónica (TCA) una triple intención: primero, cercanía con Estados vecinos; segundo, oposición frente a Estados Unidos, y tercero, protección de su territorio. Con esta celebración se fomentaba el diálogo sobre los recursos y capacidades de la Amazonía y se establecían proyectos — según Gisela Da Silva (2011, p. 19), citando a Wayne Selcher- con miras a expandir la influencia brasileña en los Estados contiguos. Desde hace poco más de un siglo, el poder militar ha sido un condicionante clave para sus aspiraciones.

Así las cosas, desde principios del siglo xx Brasil ha mantenido la intención de consolidar y salvaguardar una demografía y un territorio acorde a los objetivos de incrementar su poderío latente, para establecerse como la potencia regional en América del Sur. Para ello se ha valido de la creación de relaciones con sus pares regionales, debido a que sabe que para lograr sus objetivos los Estados vecinos son los mejores aliados (Fortuna Biato, 2009). Siguiendo la misma táctica, siempre trató de adquirir mayor poder, sin afectar su relación con Estados Unidos. Es decir, mientras la preocupación por la presencia estadounidense persistía, Brasil estrechó, cada vez más, sus relaciones con el Estado del norte.

Lo anterior fue posible gracias a la salud económica del Estado sudamericano, pero en la década de los ochenta cambió el panorama. La devaluación y la inflación en Brasil se juntaron con la crisis económica latinoamericana 
(Helfand, 1999). Remmer describió la situación en estas palabras:

América Latina estaba estancada en su peor crisis económica desde la Gran Depresión. Después de que México declarara su insolvencia financiera, a mediados de 1982, los países de la región empezaron a enfrentar problemas agudos en la prestación de servicios, relativos a los altos niveles de acumulación de deuda, con un acceso limitado a financiamiento externo. (1991, p. 778)

Es importante resaltar que en esa época la inflación afectó fuertemente a Brasil, lo que acarreaba consecuencias negativas para su estrategia. Una crisis inflacionaria repercute en la capacidad de compra de un Estado, pues debido a que su moneda se hace más barata, las importaciones le resultan más costosas. Brasil, al no contar con industrias propias, se veía obligado a adquirir del extranjero su material militar. Así que, con una inflación elevada, su poder real se veía particularmente afectado. Pero, aun sin tener en consideración el caso particular de Brasil, solventar cualquier debilidad económica se presenta — según Mearsheimer- como una necesidad primordial para cualquier Estado (2001, p. 61).

En ese orden de ideas, solucionar el tema económico pasó a ser el asunto prioritario para el Estado sudamericano. A ese aspecto se dirigieron todos sus esfuerzos; así que, finalizado el periodo dictatorial, se emplearon diferentes planes de estabilización (Neri et ál, 1999). Hasta que bajo el gobierno de Itamar Franco (1992-1995), en 1994, se instauró el llamado Plan Real. Este último, basado en los primeros pasos logrados por el expresidente CoIlor de Mello (1990-1992), logró frenar el crecimiento inflacionario (Kraychete, 1995). De este modo, para 1995, Brasil logró detener la crisis económica producida por la hiperinflación.

Incluso después de haber saneado su economía, Brasil mantiene interés en incrementar el poder latente, un hecho que responde a los términos impuestos por Estados Unidos para el sistema internacional. La posición actual del Estado norteamericano, como única potencia que puede moverse libremente alrededor del globo (Posen, 2003), le ha permitido moldear el sistema internacional con miras a sus beneficios. John Ikenberry $(2011)^{10}$ explica que Estados Unidos ha logrado limitar el constante cambio del balance de poder en el ámbito internacional, y se ha configurado, así, como hegemón. Siguiendo los argumentos de Ikenberry, el país norteamericano utiliza a su favor la pauta de no agresión ni amenaza de agresión entre países —en el marco de la ONU— para mantener el statu quo, aunque haciendo la sal-

10 Por más de ser un teórico de las relaciones internacionales de planteamientos liberales, parte de los supuestos básicos del realismo para explicar el sistema internacional. 
vedad de poder intervenir económica o militarmente en caso de que algún Estado incumpla dichos términos ${ }^{11}$.

Según lo anterior, resulta sumamente costoso para los demás Estados desarrollar su poder real, puesto que cualquier intento de desplegar como potencia militar acrecentaría la incertidumbre de Estados Unidos, lo que incitaría a este último a impedir el crecimiento del primero. En ese orden de ideas, el acto racional corresponde a incrementar el poder latente para no ser percibido como una amenaza por el Estado más poderoso. Por su parte, no acrecentar el poder latente sería contraproducente, ya que, cuando se presente la oportunidad, el Estado no va a tener cómo estructurar un poder real importante y quedará relegado frente a los que sí aprovecharon la coyuntura. Sin embargo, es necesario resaltar que la plausibilidad de un reordenamiento sistémico que permita a Brasil un ascenso como gran potencia es improbable en el corto tiempo. La disputa material por la hegemonía es una eventualidad a muy largo plazo (Lee, 2002). Así las cosas, el desarrollo de poder real y un ocasional contrapeso hacia Estados Unidos debe realizarse con prudencia, lo que corresponde al actuar del Estado suramericano.
Para Hirst (2015, p. 364), a comienzos del siglo XXI Brasil empezó a hacer uso de su creciente poder latente en favor de medidas relacionadas al soft power, para adquirir mayor campo de acción. Ejemplo de ello es la conformación del grupo BRICS, lo que le habría dado una visibilidad a nivel global como una nueva alternativa para moldear las interacciones económicas (Christensen, 2013). Lo anterior se posibilita dado que dicho grupo englobaba el $43 \%$ de la población y el $21 \%$ del PIB mundial (Reinoso, 2013). Años adelante, el contexto de la crisis económica en 2008 le permitió a Brasil reforzar su contrapeso frente a las grandes potencias. Según Guido Mantega (2014) ${ }^{12}$, el grupo BRICS estaba en las capacidades de liderar la economía mundial tras dicha debacle, teniendo en cuenta que los Estados más desarrollados no mostraron gran capacidad para solventar el declive económico.

Sin embargo, en los años posteriores a la crisis, las grandes potencias recobrarían su poder económico desmintiendo las ideas del nuevo rol del BRICS. La plataforma que tal grupo les brindó a sus miembros no logró configurarse en una herramienta útil para rebalancear el sistema. De esta manera, para los Estados que buscan alcanzar el es-

11 A través del capítulo vıl de la Carta de las Naciones Unidas, el Consejo de Seguridad —según lo estipulado en el artículo 39- "determinará la existencia de toda amenaza a la paz, quebrantamiento de la paz o acto de agresión y hará recomendaciones o decidirá qué medidas serán tomadas de conformidad con los Artículos 41 y 42 para mantener o restablecer la paz y la seguridad internacionales" (United Nations, 1945). El artículo 41 explica las sanciones económicas y comerciales y el 42 , el uso de la fuerza militar.

12 Mantega fue el ministro de Hacienda brasileño, desde 2006 hasta 2015. 
tatus de potencia (Brasil, en este caso) no son suficientes unas políticas de soft power como las emanadas a través del grupo BRICS ${ }^{13}$. La configuración del sistema internacional se transforma según las variaciones en el poder real de los Estados. Las implicaciones del poder latente son importantes únicamente para ganar autonomía con el propósito de poder sostener un poder real superior.

Regionalmente ${ }^{14}$, el Estado sudamericano discrepó de la reactivación de la Cuarta Flota (Zibechi, 2008) $)^{15}$ por parte de Estados Unidos (De Castro Neves y Spektor, 2010), de la presencia en Colombia y de su posición de frente a Cuba. Fuera de la región, Brasil denunció la falta de compromiso en temas ambientales proveniente de las potencias desarrolladas (CNN Expansión, 2009) y criticó las sanciones del Consejo de Seguridad de la ONU a Irán por su supuesto desarrollo nuclear (De Castro Neves y Spektor, 2010).

Mearsheimer (2006), analizando el futuro de China frente al poderío estadounidense, explica que, aunque un enfrentamiento militar sea improbable, los Estados más fuertes de cada región deben preocuparse por ampliar la brecha de poder con los otros Estados de la zona, así como por evitar la intromisión de alguna potencia extrarregional. En este orden de ideas, las políticas adoptadas por Brasil desde principios del siglo $\mathrm{XX}$, las respuestas a la crisis de la década de los ochenta, el establecimiento y relación del grupo BRICS, y su actuar a partir de la crisis de 2008 se pueden emplear como medidas para alcanzar los objetivos descritos por el profesor estadounidense. Pero para ello es más importante que exista un sustento en el poder real, ya que con autonomía no se moldea el sistema internacional, pero con capacidades militares sí.

\section{El ámbito nuclear}

Hoy en día la tecnología necesaria para desarrollar armamento nuclear está sobre todo al alcance de Estados menos poderosos (Mearsheimer, 1998) que, al no ser las mayores potencias globales o regionales, buscarán alterar la balanza de poder, en un comportamiento acorde al de un actor racional, mientras que las grandes potencias se esforzarán por mantener el statu quo. Ese afán de las potencias nucleares se materializó en la creación del Tratado de No Proliferación Nuclear (TNP). En el acuerdo se resalta el peligro que acarrea la posibilidad de que los Estados no nucleares adquieran capacidades atómicas para uso militar, y se señala que, al incrementarse el número de países con armamento nuclear, aumenta la probabilidad de su uso. Esto último generaría efectos

13 Para entender las políticas de los BRICs como soft power, véase Stuenkel (2016).

14 Para Wolf Grabendorff (2005, p. 13), el rol de Brasil en la región es tan importante que lo señala como el líder que tiene la capacidad de crear una identidad en Suramérica.

15 Sin embargo, es tan solo un acto administrativo, ya que dicha flota no cuenta con gran poderío real. 
catastróficos $^{16}$ (United Nations, 2014). Por esta y otras razones, el acuerdo, según lo estipulado en el artículo II de dicho tratado, lleva a los signatarios que no posean armamento nuclear a comprometerse a no desarrollarlo (United Nations, 1970).

Por supuesto, la necesidad de asegurar el cumplimiento de este compromiso requirió la creación de organismos de control que vigilaran los diferentes Estados, como lo ratifica el artículo III, en el que se designan ciertas organizaciones, como la Agencia Internacional de Energía Atómica, para que verifiquen que las plantas nucleares sean de uso pacífico (United Nations, 1970). Esta última concesión se produce debido a que el uso de la energía nuclear no es necesariamente militar. El desarrollo nuclear pacífico abriría entonces nuevos debates respecto de las centrales para este tipo de tecnologías, porque la línea que divide el proceso pacífico del desarrollo militar se hace, dados los avances tecnológicos, cada vez más permeable. En ese orden de ideas, los Estados no nucleares podrían valerse de un argumento pacifista para, paulatinamente, acercarse al desarrollo militar. Los Estados con mayor poderío en el ámbito atómico buscan anticipar esta situación para poder mantener el poder relativo existente.

En medio de este panorama, los países que no cuentan con arsenal nuclear son sometidos a una constante inspección por parte de los Estados que sí tienen tal armamento. De ese modo, los países no nucleares tendrán que buscar la manera de desarrollar ese tipo de mecanismos militares sin despertar desconfianza de las grandes potencias, o renunciar a la intención de luchar por una posición más importante en el escenario global, al menos hasta que el mismo sistema, tras un reordenamiento, le abra la posibilidad de desarrollar armamento nuclear sin correr el riesgo de enfrentarse a un poder mucho mayor. Este punto fue explicado por Mearsheimer cuando analizó las posibilidades de Taiwán frente a la amenaza china. El analista estadounidense aclaró que Taiwán debería intentar obtener capacidades nucleares, de tal manera que adquiera la capacidad de disuasión ante la amenaza de Pekín. Pero tanto China como Estados Unidos - que cumple un papel a favor de Taiwán - se opondrían a tal medida, debido a que se reduciría la brecha de poder que hoy en día los favorece (2014, p. 36).

En esa encrucijada se encuentra Brasil. El Estado sudamericano ha intentado desarrollar capacidades nucleares desde mediados del siglo Xx. En 1945 se acercó a Estados Unidos con el fin de avanzar en el sector atómico (Bocco, 1989). Gracias a un acuerdo con el país del norte, obtuvo ayuda técnica y financiera para la construcción de re-

16 Se debe señalar que para los teóricos neorrealistas el hecho de que varios Estados obtengan capacidades nucleares puede brindar mayor seguridad, puesto que cada Estado estará menos dispuesto a usar su arsenal por temor a una respuesta. 
actores a cambio de la transferencia de uranio (Grabendorff, 1987) y del apoyo durante la Segunda Guerra Mundial (Cubillos Meza, 2012). Años adelante, el discurso que recibió el nombre de "Átomos para la paz", pronunciado en 1953 por el presidente estadounidense Dwight Eisenhower (1953-1961), impulsó un nuevo acercamiento del Estado sudamericano a las potencias nucleares, ya que, desde Washington, se comenzó a brindar asistencia técnica en materia nuclear a los Estados menos desarrollados.

En el marco de esa nueva cooperación, el país norteamericano entregó tres reactores para investigación a Brasil, que fueron ubicados en universidades de diferentes regiones. Esto, si bien implicaba desventajas en cuanto al poder relativo, le permitió alcanzar nuevos avances que de manera aislada se hacían muy complicados. En el mismo camino, le brindó la posibilidad de formar científicos brasileños capaces de generar progresos propios en la materia. De hecho, fue política de Estado la preparación de ingenieros nucleares para que estos tuvieran la capacidad de construir reactores de manera independiente (Cubillos Meza, 2012). De manera simultánea, para evitar quedar ligado exclusivamente a Estados Unidos en términos de avances atómicos, Brasil se acercó a potencias europeas como Alemania y Francia. Además, la potencia sudamericana, rechazó en 1967 el TNP, ya que lo veía como un limitante en su objetivo de desarrollar capacidades atómicas propias.
A través de la cooperación con las potencias mundiales, Brasil pudo obtener una central nuclear propia, cuya construcción se encargó a la empresa norteamericana Westinghouse. Esta situación fue aprovechada por Brasil para recibir capacitación por parte de Estados Unidos, de forma tal que pudiera contar con nuevos profesionales aptos para trabajar tanto en el desarrollo de la central, como en su funcionamiento (Cubillos Meza, 2012). Así, se le dio vida a la primera central nuclear brasileña, ubicada a pocos kilómetros de Río de Janeiro. La instalación industrial fue nombrada Angra I (Milanese, 2007).

Paso seguido a la construcción de la central — que entró en funcionamiento en 1972-, Brasil intentó crear acuerdos con países diferentes a Estados Unidos, pero desarrollados nuclearmente. Tal fue el caso con Canadá y, nuevamente, con la República Federal Alemana. Con esta última se dieron los acercamientos más importantes, ya que le permitieron al Estado sudamericano el enriquecimiento de uranio (Gall, 1976) y le fueron suministradas nuevas centrales nucleares y la posibilidad de construir otros reactores de manera autónoma (Milanese, 2007). En ese impulso se planificó la construcción de Angra II para 1992 y Angra III para 1995. Pero la crisis económica retraso las obras, y Angra II fue culminada en 2000 (Cubillos Meza, 2012), mientras que la entrada en funcionamiento de Angra III está proyectada para mayo de 2018 (Electrobras, 2014). 
El esfuerzo nuclear brasileño resultó infructuoso; su atraso frente a las potencias atómicas a nivel mundial era evidente, y, peor aún, su atraso frente a su competidor regional, Argentina, también era notorio. Así que optó por adherirse a la cooperación internacional en términos nucleares ${ }^{17}$. Al contar con los elementos, tanto del poder latente como del poder real, suficientes para erguirse como hegemón de su región, se preocupó por evitar que, al no poder conseguirla, algún Estado de la zona obtuviera superioridad nuclear. En ese sentido, se puede explicar el impulso para la estructuración del Tratado de Tlatelolco. De esa manera, ha podido adquirir cooperación y conocimientos para el desarrollo de sus capacidades nucleares, desde una perspectiva pacifista que, como se explicó, puede llegar a transformarse en militar.

Concretamente, en el balance actual del sistema internacional, Estados Unidos es el único actor con la capacidad de andar libremente alrededor del globo (Posen, 2003), por lo que, desde la perspectiva de Mearsheimer, busca mantener el statu quo. De ahí que emplee su poder para - junto con las otras potencias - impedir que los demás Estados obtengan capacidades nucleares militares. En ese contexto, Brasil ha optado por firmar acuerdos con participación de organizaciones internacionales, comprometiéndose a renunciar a la proliferación nuclear de carácter militar, en aras de no desperdiciar los beneficios que, en términos de poder latente, acarrean los avances atómicos (Peña Galindo y FrassonQuenoz, 2017). Así, logró obtener la tecnología y el conocimiento para su desarrollo autónomo, y consiguió un umbral transformativo en materia tecnológica, es decir, los avances y conocimientos necesarios para convertir el poder latente en poder real.

\section{Brasil en el actual ordenamiento del sistema internacional}

Para definir el comportamiento que adopta un Estado, es necesario tener en cuenta la estructura del sistema internacional (Schroeder, 1994, p. 114). Los Estados, en su intención de alcanzar el papel hegemónico, tendrán que preocuparse por el poderío de los demás (Taliaferro, 2000). En este sentido, aquellos países que no consigan tal hegemonía deberán adoptar tácticas encaminadas a obtener el poder suficiente que les permita no poner en tela de juicio su propia supervivencia (Mearsheimer, 2001). Bajo las condiciones descritas, la polaridad del sistema internacional estará en constante cambio, aunque según la estructura dicho cambio será más o menos factible.

El ordenamiento sistémico puede ser de cuatro formas: bipolar desbalan-

\footnotetext{
17 La cooperación en el tema nuclear implica que los Estados involucrados no toman ventaja militar en ese ámbito, ya que renuncian de manera abierta a desarrollar armamento atómico, a cambio de asistencia recíproca y colaboración externa para adquirir las tecnologías necesarias para el uso pacífico de ese tipo de energía (United Nations, 1970).
} 
ceado o balanceado, y multipolar desbalanceado o balanceado. En la actualidad, existe una multipolaridad desbalanceada, con Estados Unidos como el Estado más poderoso del sistema internacional (Ferguson, 2003). Su poderío militar y latente, sumado a sus capacidades nucleares, le permiten al país norteamericano hacer presencia alrededor del globo para intervenir en pro de sus intereses (Mabee, 2004). Así, pues, el ascenso de cualquier país dependerá en gran medida de las posibilidades que logre crear a su favor con respecto a la presencia estadounidense (Hurrell, 2006).

En ese contexto se ha venido dando el crecimiento del poderío brasileño. Wolf Grabendorff (2010, p. 160) señala que ese Estado sudamericano es consciente de que debe ocupar un papel importante en el ámbito internacional, ya que cuenta con los recursos, la población y el tamaño suficiente - el poder latente- para ejercer su influencia en todo el orbe. Sin embargo, la presencia de Estados Unidos ha impedido que esa motivación sea una realidad. Lo anterior también es argumentado por pensadores constructivistas como Peter Katzenstein (2005, p. 228).

Aunque autores como David Mares (2016) indiquen que la preocupación de Brasil radica en poder articular sus intereses - como país en vía de desarrollo- en el escenario global, para lo que se abre espacio mediante el respeto a la soberanía y las normas establecidas con estrategias de soft power, los esfuerzos brasileños por establecerse como una potencia son evidentes. Su actuar —en total correspondencia a un actor racional- está dirigido a aumentar su poderío para posicionarse como el hegemón de su región. Ese comportamiento se puede evidenciar a través de su preocupación por incrementar su poderío real, pero también por el interés de alejar a Estados Unidos de su región para establecerse en ella como el hegemón. La profesora Gisela Da Silva (2011) señala que, por más que en ciertos contextos la política exterior brasileña estuviera alineada con las directrices de Estados Unidos, la potencia sudamericana siempre ha mantenido una posición independiente, sobre todo desde la década de los noventa.

En la persecución de tal objetivo, desde Brasilia se apoyó la creación del Mercado Común del Sur (Mercosur). Según Gaspare Genna y Taeko Hiroi (2007), en 1991, recién acabada la Guerra Fría, el Estado sudamericano impulsó el establecimiento del Mercosur como una herramienta para establecerse como el líder en la región. Al respecto, los autores señalan que, al ser el Estado con la economía más grande del acuerdo, adquiere la posibilidad tanto de obtener mayores beneficios en negociaciones extra regionales, como de recibir un respaldo más amplio en asuntos internacionales. Para Andy Klom (2003, p. 355), ese desbalance en términos de poderío latente a favor de Brasilia en el Mercosur genera que los otros Estados pertenecientes al acuerdo aumenten 
su dependencia respecto a Brasil. En términos de Susanne Gratius (2008, p. 142), el Mercosur ha avanzado considerablemente y le ha servido a Brasil para liderar la región sudamericana.

El comportamiento del Estado lusoparlante, encaminado a abrirse el campo necesario dentro de su región para convertirse en el hegemón sudamericano, ha cobrado mayor relevancia, debido a su alejamiento de la potencia del norte. Si bien Sudamérica ha sido dominada, desde años atrás, por Estados Unidos, la zona —salvo durante la Segunda Guerra Mundialha tenido un limitado valor estratégico para Washington (Soares y Hirst, 2006, p. 22). Esta situación cobró mayor importancia después de los atentados del 11 de septiembre del 2001 (Pastrana Buelvas, 2009), eventualidad que ha sido aprovechada por Brasilia para incrementar su capacidad militar.

Aun así, en el entorno de un sistema multipolar desbalanceado, la opción brasileña de configurarse como hegemón regional ha venido siendo limitada por el poderío superior de Estados Unidos (Weyland, 2016). El país norteamericano se ha encargado de hacer presencia mundial con la intención de mantener el statu quo y así evitar que crezcan nuevas potencias capaces de poner en consideración su posición privilegiada. Mearsheimer (1990, p. 15) advierte que en un sistema multipolar desbalanceado se dificulta disuadir a la gran potencia, así que Brasil ha tenido que ser cauteloso a la hora de desarrollar un poder real que le permi- ta erguirse como "un actor internacional a tener en cuenta por las grandes potencias en el concierto de las naciones" (Runza, 2008, s. p.); papel que, según lo descrito hasta este punto, ha buscado mediante el incremento de sus capacidades materiales.

\section{Conclusión}

Desde la óptica de los enunciados expuestos por John Mearsheimer, Brasil, a partir de 1985, ha tenido que hacer frente al potencial estadounidense para poder adoptar políticas encaminadas a aumentar su poderío militar, con el objetivo de establecerse como hegemón regional. Para ello, ha sido necesario que el Estado sudamericano desarrolle capacidades latentes capaces de sostener e impulsar los avances en materia de defensa. Entendiendo que, con dicho poder, el latente, puede ganar autonomía, pero no moldear la polaridad del sistema, cuestión contraria a los planteamientos del soft power.

Al mismo tiempo, para Brasil ha sido importante su relación con el Estado norteamericano, pero también con otras potencias, y es importante que tales potencias no detengan su crecimiento. Es en ese sentido, el país suramericano no ha podido incrementar su poder real de manera abierta, sino que se ha visto obligado a hacerlo sin despertar incertidumbre (Peña Galindo y Frasson-Quenoz, 2017). Es necesario recordar que, desde el realismo estructural, el poder militar no necesariamente debe ser empleado para 
lograr una reconfiguración del sistema internacional.

La capacidad real de los Estados se puede enmarcar en un ámbito disuasivo (Zagare, 1987; Mearsheimer, 2001). Así las cosas, aunque Brasil no haya demostrado intenciones de utilizar sus capacidades materiales, el hecho de fortalecerlas y ganar autonomía en ese ámbito es suficiente para que su poderío militar entre en consideración, con miras a un cambio a su favor en la arquitectura sistémica.

Por lo anterior, Brasil ha desarrollado - con la cautela necesaria - las bases para un poder real futuro. En ese sentido, ha establecido cooperación estratégica con potencias europeas y avances propios, para reforzar sus capacidades materiales. Ejemplo de lo anterior es el inicio de la construcción de cuatro submarinos militares que empezarían a funcionar entre 2018 y 2022 (Barreira, 2017).

No obstante, la situación de no contar con armamento nuclear se configura como su obstáculo más grande, puesto que ello le otorga superioridad a los Estados que cuentan con tal tipo de instrumentos. De esta forma, Brasil se ha preocupado por impedir que otro Estado de su región incremente su poderío real con instrumentos atómicos.

Al mismo tiempo, ha procurado desarrollar avances en materia nuclear sin despertar alertas en los países más poderosos del sistema. Para ello, ha apoyado a diferentes Estados que en contraposición a Estados Unidosbuscan desplegar industria nuclear propia, bajo el argumento de que sus intenciones no son bélicas.

Con esa misma línea argumentativa, la potencia sudamericana ha venido adquiriendo tanto los conocimientos, como los instrumentos para controlar el proceso nuclear, de tal manera que pueda producir ese tipo de armamento cuando lo considere oportuno y así establecerse como una gran potencia en el escenario internacional.

Dicha táctica cumplió sus objetivos hasta 2012, pero después de que las grandes potencias empezaron a solventar sus crisis financieras, el poder latente relativo de Brasil volvió a retroceder.

Ese escenario traza nuevos retos para el Estado sudamericano, el cual debe replantear sus políticas, con miras a no quedar relegado frente los países más poderosos, que al parecer están tomando un nuevo aire. Todo esto, teniendo en mente que el soft power no le brindará seguridad en un sistema internacional anárquico.

\section{Referencias}

Acuña, C., Smith, W. y Wolfson, L. (1994). Política y "economía militar" en el cono sur: democracia, producción de armamentos y carrera armamentista en la Argentina, Brasil y Chile. Desarrollo económico, 34(135), 343-378. 
Amorim, C. (2012). Estrategia de defensa de Brasil y de América del Sur. Revista de Ciencia Política, 32(2), 501-507.

Argumosa, J. (2011). Brasil camino de Gran Potencia. Documentos de Seguridad y Defensa, (40) 13-30.

Banco Mundial. (2005). Reseña sobre Brasil. Recuperado de http://web. worldbank.org/WBSITE/EXTERNAL/ BANCOMUNDIAL/NEWSSPANISH/0, C ontentMDK:20753788 pagePK: 64257043 piPK:437376 theSite PK:1074568,00.html

Barreira, V. (2017), LAAD 2017: Brazilian submarine programme moves into construction. Recuperado de http:// www.janes.com/article/69452/ laad-2017-brazilian-submarineprogramme-moves-into-construction?utm_campaign $=$ PC6110 E $17 \% 20$ D F \% $20 \mathrm{NL} \% 2 \overline{0}$ Naval\%2018_04_17\&utm_ m e dium $=$ e ma i I \& u t m _ source $=$ Eloqua

Bertonha, J. F. (2008). El poder militar brasileño y sus dilemas en el viraje del Siglo xxı. Temas del Cono Sur Dossier Integración, (44), 3-8.

Bertonha, J. F.(2010a). Adquisiciones de armamentos y reequilibrios geopolíticos: América del Sur en la primera década del siglo xxı. Revista de Relaciones Internacionales de la Universidad Nacional de la Plata, (39), 109-127.

Bertonha, J. F. (2010b), Brazil: an emerging military power? The problem of the use of force in Brazilian international relations in the 21st century. Revista Brasilera de Politica Internacional, 53(2), 107-124.

Bocco, H. (1989), La cooperación nuclear Argentina-Brasil. Notas para una evaluación política. Buenos Aires: Facultad Latinoamericana de Ciencias Sociales (Flacso).

Buzan, B. y Waever, O. (2003). Regions and Powers. Cambridge: Cambridge University Press.

Calle, F. (2008). La geopolítica sudamericana de Brasil en el siglo xxı. Colección, (19), 167-180.

Candeas, A. (2012). Brasil y Colombia: vecinos otrora distantes descubren el potencial de su relación. En E. Pastrana, Jost, E. y Flemes, D. (eds.), Colombia y Brasil: ¿socios estratégicos en la construcción de Suramérica? (pp. 283-307). Bogotá: Editorial Pontificia Universidad Javeriana.

Chatin, M. (2016). Brazil: analysis of a rising soft power. Journal of Political Power, 9(3), 369-393.

Christensen, S. F. (2013). Brazil's Foreign Policy Priorities. Third World Quarterly, 34(2), 271-286.

Central Intelligence Agency (CIA). (2013). cIA World Factbook. Recuperado de https://www.cia.gov/ library/publications/the-world-factbook/geos/br.html

Costa, H. (2007). Jobim diz que reaparelhamento militar é necessário e descarta corrida armamentista. AgenciaBrasil. Recuperadodehttp:// memoria.ebc.com.br/agenciabrasil/noticia/2007-10-31/ jobim-diz-que-reaparelhamento- 
militar-e-necessario-e-descarta-corrida-armamentista

Cubillos, A. (2012). El desarrollo nuclear de Brasil: reseña histórica. Memorias, 9(17), 170-204.

Da Silva, G. (2011). Brasil, opciones estratégicas de una potencia emergente para afirmar su liderazgo mundial. Oasis, 16(1), 5-23.

De Castro, J. y Spektor, M. (2010). Obama, Lula y la relación entre los Estados Unidos y Brasil. En A. Lowenthal, T. Piccone y L.Whitehead (eds.), Obama y las Américas: iesperanza o decepción? (pp. 79-97). Bogotá: Planeta.

Delgado, J. (2011). Brasil: la emergencia de la región amazónica en la defensa nacional. Konrad Adenauer Stiftung. Recuperado de http:// www.kas.de/wf/doc/4928-1442-430.pdf

Duarte, R. y Viana, M. (2008). Política exterior brasileña: nuevos y viejos caminos en los aspectos institucionales, en la práctica del multilateralismo y en la política para el Sur. Revista de Ciencia Política, 28(2), 77-106.

Electrobras. (2014). Angra 3: energia para o crescimento do país. Recuperado de http://www.eletronuclear. gov.br/aempresa/centralnuclear/angra3.aspx

Ferguson, N. (2003). Power. Foreign Policy, (134), 18-22.

Fernández, T. (2007). José María Da Silva Paranhos, Barón de Río Bran- co. Culminación de una exitosa política de límites. Epocas, (1), 125143.

Flemes, D. (2012). Actores estatales y regionalismo estratégico: Brasil y Colombia en el orden multipolar. En Pastrana, E., Jost, E. y Flemes, D. (eds.), Colombia y Brasil: ¿socios estratégicos en la construcción de Suramérica? (pp. 25-50). Bogotá: Editorial Pontificia Universidad Javeriana.

Fondo Monetario Internacional. (2010). World Economic Outlook database. Recuperado de http://www.imf.org/ external/pubs/ft/weo/2010/02/map/

Fortuna Biato, M. (2009). La política exterior de Brasil: iintegrar o despegar? Estudios de Política Exterior. Recuperado de http://www.politicaexterior.com/archives/5831/comment-page- 1

Fresard, C. y Olarte, A. M. (2001). Indicadores macroeconómicos de América Latina. 1990-2000. Lecturas de Economía, (54), 151-165.

Gall, N. (1976). Atoms for Brazil, Dangers for All. Foreign Policy, (23), 155-201.

Genna, G. y Hiroi, T. (2007). Brazilian regional power in the development of Mercosul. Latin American Perspectives, 34(5), 43-57.

González, Á. (2003). Presente y futuro de las relaciones civico-militares en Hispanoamerica. Instituto Español de Estudios Estratégicos. Recuperado de http://www.ieee.es/Galerias/ fichero/cuadernos/CE_123_Rela- 
cionesCivicoMilitaresHispanoamerica.pdf

Goyzueta, V. (8 de septiembre de 2009). Brasil anuncia la mayor compra de armamento militar de su historia. $A B C$ de España.

Grabendorff, W. (1987). La política nuclear y de no proliferación de Brasil. Estudios Internacionales, 20(80), 520-568.

Grabendorff, W. (2005). Relaciones triangulares en un mundo unipolar: América del Norte, la Unión Europea y América del Sur. Análisis Político, 18(55), 3-19.

Grabendorff, W. (2010). Brasil: de coloso regional a potencia global. Nueva Sociedad, (226), 158-171.

Gratius, S. (2008). Brasil emerge como potencia regional y global. Quórum, (22), 135-146.

Helfand, S. (1999). The political economy of agricultural policy in Brazil: Decision making and influence from 1964 to 1992. Latin American Research Review, 34(2), 3-41.

Hepple, L. (1986). Geopolitics, generals and the state in Brazil. Political Geography Quarterly, 5(4), 579590.

Hirst, M. (2015). Emerging Brazil: The Challenges of Liberal Peace and Global Governance. Global Society, 29(3), 359-372.

Hurrell, A. (2006). Hegemony, Liberalism and Global Order: What Space for Would-Be Great Powers? International Affairs, 82(1), 1-19.
Ikenberry, J. (2011). Liberal leviathan: the origins, crisis, and transformation of the american world order. Nueva Jersey: Princeton University Press.

Kaplan, M. (1957). System and Process in International Politics. Nueva York: Wiley.

Kapstein, E. (1990). The brazilian defense industry and the international syste. Political Science Quarterly, 105(4), 579-596.

Katzenstein, P. (2005). A world of regions. Nueva York: Cornell University Press.

Klom, A. (2003). Mercosur and Brazil: A european perspective. International Affairs, 79(2), 351-368.

Kraychete, G. (1995). El "Plan Real" de Brasil y las trampas de las políticas de estabilización en América Latina. Fomento Social, (199), 337396.

Lee, G. (2002). To be long or not to be long-that is the question: The contradiction of time-horizon in offensive realism. Security Studies, 12(2), 196-217.

Lula critica promesa de EU en Copenhague. (2009). CNN Expansión. Recuperado de http:// www.cnnexpansion.com/actuali$\mathrm{dad} / 2009 / 12 / 21 /$ lula-critica-promesa-de-eu-en-copenhague

Mabee, B. (2004). Discourses of Empire: The US 'Empire', Globalisation and International Relations. Third World Quarterly, 25(8), 1359-1378. 
Mantega, G. (2014). Foro Económico Mundial 2014. Recuperado de https://www.youtube.com/ watch? $v=$ R0Ks7EosHME

Mares, D. (2016), Brazil Revising the status quo with soft power? En T.V. Paul (Ed.), Accommodating Rising Powers (pp. 246-267). Cambridge: Cambridge University Press.

Mearsheimer, J. (1990). Back to the Future: Instability in Europe after the Cold War. International Security, 15(1), 5-56.

Mearsheimer, J. (17 de mayo de 1998). Here We Go Again. The New York Times.

Mearsheimer, J. (2001). The Tragedy of Great Power Politics. Nueva York: Norton.

Mearsheimer, J. (2006). China's Unpeaceful Rise. Current History, 105(690), 160-162.

Mearsheimer, J. (2009). Reckless States and Realism. International Relations, 23(2), 241-256.

Mearsheimer, J. (2014). Taiwan's Dire Straits. The National Interest, 130(2), 29-39.

Mercado-Jarrín, E. (1982). Armamentismo en América Latina y reducción de los gastos militares. Nueva Sociedad, (59), 5-22.

Milanese, J. P. (2007). Uso pacífico de la energía nuclear. Cali: Universidad ICESI.

Ministerio de Defensa de Brasil. (2008). Defesa em comitiva oficial no Suriname reafirma acordos de cooperação. Recuperado de http:// www.defesa.gov.br/projetosweb/ estrategia/arquivos/estrategia_defesa_nacional_espanhol.pdf

Moniz Bandeira, L. A. (2006). Brazil as a Regional Power and its Relations With the United States. Latin American Perspectives, 33(3), 12-27.

Neri, M., Pinto Carvalho, A., Abrahão de Castro, J., Datrino, M. F. et al. (1999). Gasto Público en servicios sociales básicos en América Latina y el Caribe. Brasil. CEPAL. Recuperado de http://www.eclac.org/cgi-bin/ getProd.asp?xml=/publicaciones/ $\mathrm{xml} / 8 / 4648 / \mathrm{P} 4648 . x \mathrm{ml} \& \mathrm{xs}=/ \mathrm{tpl} /$ p9f.xsl\&base $=/$ tpl/top bottom.xsl

Organización de las Naciones Unidas. (1945). Carta de las Naciones Unidas. Recuperado de http://www. un.org/es/charter-united-nations/ index.html

Organización de las Naciones Unidas. (1970). Treaty on the Non-Proliferation of Nuclear Weapons. Recuperado de http://disarmament.un.org/ treaties/t/npt/text

Organización de las Naciones Unidas. (2014). Nuclear Weapons. Recuperado de http://www.un.org/disarmament/WMD/Nuclear/

Pastrana Buelvas, E. (12 de septiembre de 2009). Brasil se arma; su capacidad militar cambiará sustancialmente tras acuerdo con Francia. El Tiempo.

Pastrana Buelvas, E. y Vera Piñeros, D. (2012). Rasgos de la política exterior brasileña en su proceso de ascenso como potencia regional y 
jugador global. En E. Pastrana, Jost, S. y Flemes, D. (Eds.), Colombia y Brasil: isocios estratégicos en la construcción de Suramérica? (pp. 135-184). Bogotá: Editorial Pontificia Universidad Javeriana.

Peña, A. y Frasson-Quenoz, F. (2017). Brasil: ¿hegemonía a pesar de todo? Bogotá: Universidad Externado de Colombia.

Poggio, C. (2014). Brazil, The United States, and the South American Subsystem. Plymouth: Lexington Books.

Posen, B. (2003). Command of the Commons. The Military Foundation of U.S. Hegemony. International Security, 28(1), 5-46.

Reinoso, J. (26 de marzo de 2013). Los emergentes exigen su cuota de poder. El País. Recuperado de https://elpais.com/internacional/2013/03/26/ actualidad/1364326329_993675. html

Remmer, K. (1991). The Political Impact of Economic Crisis in Latin America in the 1980s. The American Political Science Review, 85(3), 777-800.

Red de Seguridad y Defensa de América Latina (Resdal). (2012). Atlas Comparativo de la Defensa en América Latina y el Caribe. Recuperado de http://www.resdal.org/atlas/atlas-libro-12-espanol.html

Runza, R. (2008). La construcción de una Comunidad de Seguridad en América del Sur a la luz de la adquisición de armamento. Policy Pa- per 20, 1-8. Recuperado de http:// library.fes.de/pdf-files/bueros/la-seguridad/50503.pdf

Schroeder, P. (1994). Historical Reality vs. Neo-Realist Theory. International Security, 19(1), 108-148.

Stockholm International Peace Research Institute (Sipri) (1978). World Armaments and Disarmament: SIPRI Yearbook 1978. Washington: Stockholm International Peace Research Institute.

Stockholm International Peace Research Institute (Sipri) (1982). World Armaments and Disarmament: SIPRI Yearbook 1982. Washington: Stockholm International Peace Research Institute.

Stockholm International Peace Research Institute (Sipri) (2003). Armaments, Disarmament and International Security: SIPRI Yearbook 2003. Nueva York: Oxford University Press.

Stockholm International Peace Research Institute (Sipri) (2011). Armaments, Disarmament and International Security: SIPRI Yearbook 2011. Nueva York: Oxford University Press.

Soares, M. y Hirst, M. (2006). Brazil as an Intermediate State and Regional Power: Action, Choice and Responsibilities. International Affairs (Royal Institute of International Affairs 1944-), 82(1), 21-40.

Taliaferro, J. (2000). Security Seeking under Anarchy: Defensive Rea- 
lism Revisited. International Security, 25(3), 128-161.

Tokatlian, J. (2011). Latinoamérica y sus opciones estratégicas: un análisis de las relaciones extra-regionales. Análisis Político, 24(73), 139-158.

Uría, I. (2011). Brasil se arma. Antiguos Alumnos del IEEM, (1), 15-16.

Vargas Vergnaud, M. (2004). Una mirada económica a los acuerdos de offsets en el Sector Defensa y Seguridad en Colombia. Bogotá: Departamento Nacional de Planeación.

Vitelli, M. (2016). La identidad estratégica argentina y el ascenso de Brasil. Las bases ideacionales de una política de defensa cooperativa. Revista de Relaciones Internacionales, Estrategia y Seguridad, 11(2), 271-289.
Weyland, K. (2016). Realism under Hegemony: Theorizing the Rise of Brazil. Journal of Politics in Latin America, 8(2), 143-173.

Zagare, F. (1987). The Dynamics of Deterrence. Chicago: University of Chicago Press.

Zibechi, R. (9 de mayo de 2008). Cuarta Flota: mensaje de guerra. La Jornada. Recuperado de http:// www.jornada.com.mx/2008/05/09/ index.php?section $=$ opinion \&articl $\mathrm{e}=024 \mathrm{a} 2 \mathrm{pol}$

Zurbriggen, C. (2005). Política exterior, defensa y las operaciones de paz: ¿una estrategia coherente? El caso de Uruguay. Revista Fuerzas Armadas y Sociedad, 19(1), 85-109.

Zweig, S. (2012). Brasil, país de futuro. Madrid: Capitan Swing. 
